TIFR/TH/00-44

August 2000

\title{
A Note on Gauge Invariant Operators in Noncommutative Gauge Theories and the Matrix Model
}

\author{
Avinash Dhar and Spenta R. Wadia \\ Department of Theoretical Physics, \\ Tata Institute of Fundamental Research, \\ Homi Bhabha Road, Mumbai 400 005, INDIA. \\ adhar@tifr.res.in,wadia@tifr.res.in
}

\begin{abstract}
In this note we discuss local gauge-invariant operators in noncommutative gauge theories. Inspired by the connection of these theories with the Matrix model, we give a simple construction of a complete set of gauge-invariant operators. We make connection with the recent discussions of candidate operators which are dual to closed strings modes. We also discuss large Wilson loops which in the limit of vanishing noncommutativity, reduce to the closed Wilson loops of the ordinary gauge theory.
\end{abstract}




\section{Introduction}

One question of intrinsic interest in any gauge theory is the construction of local gaugeinvariant operators since these form a complete set of observables of the theory. This question has been addressed in several recent works 11, 2, 3, 4, 5, 6, 0, 8, 9, 10, 11 in the context of noncommutative gauge theories. In these theories the construction of gauge-invariant operators is made somewhat nontrivial by the fact that simple analogues of local gaugeinvariant operators of commutative gauge theories, taken over to the noncommutative case, are not local, but are integrated over all of space. It turns out, however, that more general gauge-invariant operators do exist and were constructed in [7]. The set of gauge-invariant operators in noncommutative gauge theories constructed in this work has been further discussed

in [13, 14, 15]. Furthermore, operators presented in [15] have the desirable property that they reproduce the corresponding local operators in ordinary commutative gauge theories at scales large compared to the noncommutativity scale.

In this note we exploit the connection [16, 17, 18, 19, 20, 21, 7, 23, 22, 8] of the matrix model to noncommutative gauge theories (emphasized recently by Seiberg 24]) to present a particularly simple construction of local gauge-invariant operators in these theories. The matrix model has an underlying $U(N)$ gauge symmetry and it is easy to write operators invariant under this symmetry. Dp-branes arise in the matrix model as classical solutions in the limit of large $N$ and the $U(N)$ gauge symmetry of the matrix model reappears on the branes as the noncommutative gauge symmetry. Because of this connection $U(N)$-invariant operators of the matrix model reappear as gauge-invariant operators on the branes. This fact directly leads to a simple construction of local gauge-invariant operators in noncommutative gauge theories.

\section{Dp-branes in Matrix Model}

The dynamical variables in the matrix model 25] are nine $N \times N$ hermitian matrices $X^{I}$, $I=1,2, \ldots, 9$. Time-independent bosonic classical solutions are determined by extremizing the potential $\operatorname{Tr}\left[X^{I}, X^{J}\right]^{2}$, which gives the equation of motion

$$
\left[X_{J},\left[X^{I}, X^{J}\right]\right]=0
$$

In the large $\mathrm{N}$ limit Dp-branes correspond to the solutions of this equation given by [16]

$$
\begin{aligned}
& X^{i}=x^{i}, i=1,2, \ldots, p \\
& X^{I}=0, I=p+1, \ldots, 9 .
\end{aligned}
$$

where

$$
\left[x^{i}, x^{j}\right]=i \theta^{i j}
$$

The rank of the matrix $\theta$ is $p$ for Dp-branes so that one has maximal noncommutativity. Also note that in the matrix model $p$ is even. 
Expanding $X^{I}$ around the above classical solution gives a noncommutative gauge theory on the branes. If the classical solution represents $Q$ Dp-branes, one gets a $U(Q)$ noncommutative gauge theory.

Let us parameterize the fluctuations as

$$
X^{i}=x^{i}+\theta^{i j} \hat{A}_{j}\left(x^{i}\right)
$$

We have written the fluctuations as dependent on $x^{i}$ because we can expand it in terms of a complete set of operators. An example, in the large $\mathrm{N}$ limit, is the Weyl basis defined by the exponential operators $g(\alpha)=\exp i \alpha_{i} x^{i}$. We also have the scalar fluctuations $\phi^{a}=X^{a+p}, a=$ $1,2, \ldots, 9-p$.

Now, the time independent $U(N)$ gauge symmetry of the matrix model $]$ acts on $X^{I}$ as $X^{I} \rightarrow U X^{I} U^{\dagger}$. This action descends on the fluctuations $\hat{A}_{i}$ on the branes as $U(Q)$ noncommutative gauge symmetry

$$
\hat{A}_{i} \rightarrow U \hat{A}_{i} U^{\dagger}+i U \bar{\partial}_{i} U^{\dagger}
$$

where

$$
\bar{\partial}_{i}=-i \theta_{i j}^{-1} a d x_{j}
$$

provided we transfer, as above, the $U(N)$ transformation of the background to the fluctuation. Here $\theta^{-1}$ is the inverse of the matrix $\theta$.

The transformation of the fluctuation in (5) is indeed a gauge transformation because the second term can be understood as a parallel transport [11, 10] defined in terms of the derivative operator of the non-commutative gauge theory. From here it is clear that the gauge group of the non-commutative gauge theory is inherited from the large $\mathrm{N}$ limit of the matrix model, and also the definition (6) makes it clear that translations are generated by $U(\infty)$ rotations. Thus, we see that the set of gauge-invariant operators on the branes is identical to and directly inherited from the gauge-invariant operators in the matrix model. Also gauge invariant operators are necessarily translation invariant.

\section{$3 \quad$ Gauge Invariant Operators}

Gauge-invariant operators are very easy to construct in the matrix model because its $U(N)$ gauge symmetry is linearly realized on the dynamical variables $X^{I}$. Thus, the matrix trace of an arbitrary function of $X^{I}$ is gauge-invariant. A complete set of gauge-invariant operators is given by $\operatorname{Tr}\left(X^{I_{1}} \ldots X^{I_{n}}\right)$ where $n \leq N$, since for $n \geq N$ the trace can be rewritten in terms of traces of fewer number of matrices. In the large $N$ limit, which is what we need in the Dp-brane classical background, it is more convenient to consider the operators

$$
\hat{O}_{k}=e^{i k \cdot X} .
$$

\footnotetext{
${ }^{1}$ In the IKKT matrix model 26 this will be the full gauge symmetry
} 
Traces of products of these operators for different values of $k$ can be used to generate all the above gauge-invariant operators for finite $N$. For example,

$$
\operatorname{Tr}\left[X^{I}, X^{J}\right]^{2}=-\left.2\left(\partial_{I} \partial^{\prime}{ }_{J}-\partial_{J} \partial_{I}^{\prime}\right)^{2} \operatorname{Tr}\left(e^{i k \cdot X} e^{i k^{\prime} \cdot X}\right)\right|_{k=k^{\prime}=0},
$$

where $\partial$ and $\partial^{\prime}$ are derivatives with respect to $k$ and $k^{\prime}$. That is, all the gauge-invariant operators of the matrix model are contained in the set of operators

$$
\hat{O}_{k k^{\prime} \ldots}=e^{i k \cdot X} e^{i k^{\prime} \cdot X} \ldots
$$

By the correspondence of the matrix model $U(N)$ gauge symmetry with the noncommutative gauge symmetry on the branes discussed above, then, this set of operators must reproduce all the gauge-invariant operators in the noncommutative gauge theory on the branes. In the rest of this note we will show that this is indeed the case.

\subsection{Straight and Curved Wilson lines}

Traces of operators of the type in (7) can be given the interpretation of straight Wilson lines in the noncommutative gauge theory on the branes. To see how that comes about, let us write

$$
\begin{aligned}
\hat{O}_{k} & =e^{i k \cdot X} \\
& =e^{i \frac{k}{n} \cdot X} e^{i \frac{k}{n} \cdot X} \cdots(n \text { factors }) .
\end{aligned}
$$

At the end we will take the limit $n \rightarrow \infty$. Here we will restrict ourselves to $k_{I}$ with nonzero components only in the brane directions. The more general case is treated in the next sub-section. Let us now use (田) to write, in an obvious notation,

$$
\begin{aligned}
e^{i \frac{k}{n} \cdot X} & =e^{i \epsilon \theta^{-1} \cdot x+i \epsilon \cdot \hat{A}(x)} \\
& =e^{i \epsilon \theta^{-1} \cdot x} e^{i \epsilon \cdot \hat{A}(x)+o\left(\epsilon^{2}\right)}
\end{aligned}
$$

where $\epsilon=\frac{k}{n} \theta$. Using this in (9), we get

$$
\begin{aligned}
\hat{O}_{k} & =e^{i \epsilon \theta^{-1} \cdot x} e^{i \epsilon \cdot \hat{A}(x)+o\left(\epsilon^{2}\right)} e^{i \epsilon \theta^{-1} \cdot x} e^{i \epsilon \cdot \hat{A}(x)+o\left(\epsilon^{2}\right)} \cdots(n \text { factors }) \\
& =e^{i \epsilon \cdot \hat{A}(x+\epsilon)+o\left(\epsilon^{2}\right)} e^{i \epsilon \cdot \hat{A}(x+2 \epsilon)+o\left(\epsilon^{2}\right)} \cdots e^{i \epsilon \cdot \hat{A}(x+n \epsilon)+o\left(\epsilon^{2}\right)} e^{i n \epsilon \theta^{-1} \cdot x}
\end{aligned}
$$

In arriving at the last line above we have used the fact that the adjoint action of $x$ generates a translation by virtue of the algebra (3). In the limit $n \rightarrow \infty$, the product of operators involving the gauge field in the last line gives the Wilson line operator $U(x, x+k \theta)$ along a straight line path given by the vector $k \theta$. Thus the gauge-invariant operator $\operatorname{Tr} \hat{O}_{k}$ of the matrix theory translates into the Wilson line $\operatorname{Tr} U(x, x+k \theta) e^{i k \cdot x}$ in the noncommutative gauge theory on the branes.

Upto a phase, the more general operators $\hat{O}_{k k^{\prime} \ldots}$ given in (8) can similarly be interpreted in the noncommutative gauge theory on the branes as Wilson line operators along a general curved path determined by the straight line segments given by the vectors $k \theta, k^{\prime} \theta$, etc. That is,

$$
\hat{O}_{k k^{\prime} \ldots}=U(x, x+k \theta) U\left(x+k \theta, x+\left(k+k^{\prime}\right) \theta\right) \cdots e^{i k \cdot x} e^{i k^{\prime} \cdot x} \cdots
$$


An example of such a Wilson line has been shown in Fig. 1. Since any continuous curve can be approximated by straight line segments one can easily construct the Wilson line corresponding to an arbitrary open curve.

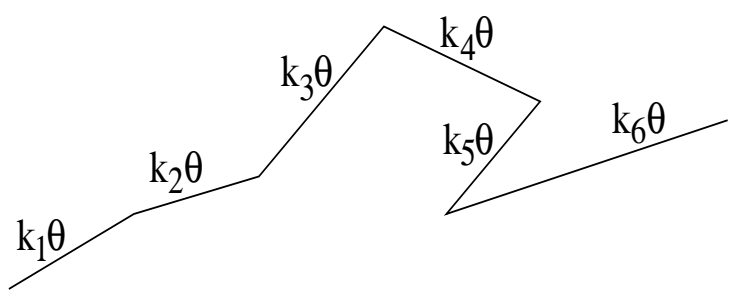

Fig. 1: Polygonal Wilson line

Note that if $x_{i}$ is a brane background then so is $x_{i}+a_{i} I$ where $I$ is the identity operator and $a_{i}$ is a real number. This fact immediately implies that the expectation value of $\operatorname{Tr} \hat{O}_{k k^{\prime}} \ldots$ conserves momentum and is non-zero only if the sum $k+k^{\prime}+\ldots=0$.

\subsection{Generalised Wilson Lines}

Let us now consider the more general situation of arbitrary $k_{I}$, i.e. $k_{I}=\left(k_{i}, s_{a}\right)$ where $i=1,2, \ldots, p$ and $a=1,2, \ldots, 9-p$. In this case proceeding after eqn. (9), we have

$$
e^{i \frac{k}{n} \cdot X}=e^{i \epsilon \theta^{-1} \cdot x+i \epsilon^{i} \hat{A}(x)_{i}+i \frac{s a}{n} \hat{\phi}_{a}(x)}
$$

where as before $\epsilon^{i}=\frac{k_{j}}{n} \theta^{j i}$. Thus the analogue of (10) is

$$
\begin{aligned}
\hat{O}_{k}= & e^{i \epsilon^{i} \hat{A}_{i}(x+\epsilon)+i \frac{s_{a}}{n} \hat{\phi}_{a}(x+\epsilon)+o\left(\frac{1}{n^{2}}\right)} e^{i \epsilon^{i} \hat{A}_{i}(x+2 \epsilon)+i \frac{s_{a}}{n} \hat{\phi}_{a}(x+2 \epsilon)+o\left(\frac{1}{n^{2}}\right)} \\
& \cdots e^{i \epsilon^{i} \hat{A}_{i}(x+n \epsilon)+i \frac{s a}{n} \hat{\phi}_{a}(x+n \epsilon)+o\left(\frac{1}{n^{2}}\right)} e^{i n \epsilon \theta^{-1} \cdot x}
\end{aligned}
$$

In the limit $n \rightarrow \infty$ this gives rise to a modified Wilson line operator $U_{s}(x, x+k \theta)$ along the straight line path given by the vector $k \theta$. The path is now characterized by the additional 'internal' quantities $s_{a}, s_{a}^{\prime}, \ldots$. These generalized Wilson line operators are similar to those introduced by Maldacena [12] in the context of AdS/CFT. It would be nice to understand the connection more quantitatively.

\subsection{Operators Dual to the Closed String Modes}

When the sum of momenta $\left(k+k^{\prime}+\cdots\right)$ vanishes, the operator $\hat{O}_{k k^{\prime} \ldots}$ corresponds, on the branes, to a closed Wilson loop (untraced), which was defined e.g. in [11] with $\epsilon_{i}^{m}=\left(k^{m} \theta\right)_{i}$ 
being the length of the mth loop segment. One can let $m \rightarrow \infty$ and $k^{m} \rightarrow 0$ for fixed $\theta$ in such a way that the polygonal loop becomes a continuous curve with no net momentum. Of course, in general one also includes here operators that traverse the geometrical loop several times.

Now, a special class of operators arises when an operator, which corresponds to one or more closed Wilson loops, is present anywhere in the product of exponentials that defines the general operator $\hat{O}_{k k^{\prime} \ldots}$. On the branes, such an operator corresponds to a Wilson line with a closed polygon loop somewhere along it. An example of such a Wilson line with a single closed loop is shown in Fig 2. In the case of a single closed loop, the position of the loop along the Wilson line does not matter. This is because we are dealing with the trace of the operator (8) in which a certain sequence of momenta adds up to zero.

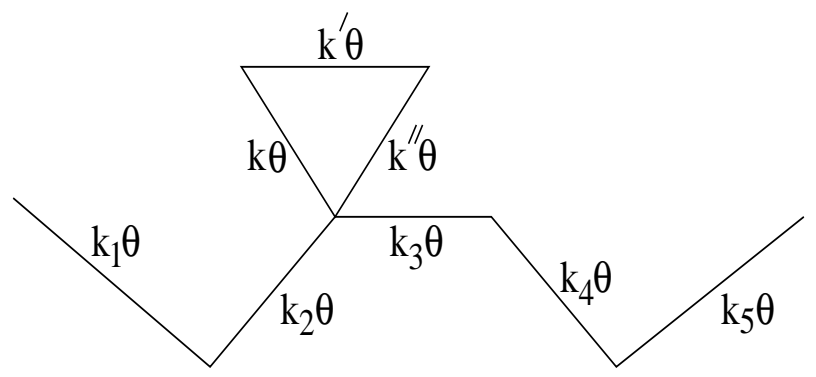

Fig. 2: Wilson line with an attached closed loop

The above constructions are easily generalized to continuous curves. If the open curve is a straight line specified by a fixed momentum and the closed loop is vanishingly small, the Wilson line generates the gauge-invariant operators that have recently been discussed in [15] as candidate operators dual to supergravity modes. As an example, consider the following operator

$$
\hat{O}_{k_{1} k k^{\prime} k^{\prime \prime} k_{2}}=e^{i k_{1} \cdot X} e^{i k \cdot X} e^{i k^{\prime} \cdot X} e^{i k^{\prime \prime} \cdot X} e^{i k_{2} \cdot X}
$$

where $\left(k+k^{\prime}+k^{\prime \prime}\right)=0$ and $k$ and $k^{\prime}$ are small, though $k_{1}$ and $k_{2}$ need not be small. Writing $k^{\prime \prime}=-\left(k+k^{\prime}\right)$ we have

$$
e^{i k \cdot X} e^{i k^{\prime} \cdot X} e^{-i\left(k+k^{\prime}\right) \cdot X}=1-\frac{1}{2}\left[k \cdot X, k^{\prime} \cdot X\right]+O\left(k^{3}\right)
$$

Thus we get

$$
\hat{O}_{k_{1} k k^{\prime} k^{\prime \prime} k_{2}}=e^{i k_{1} \cdot X} e^{i k_{2} \cdot X}-\frac{1}{2} k_{I} k_{J}^{\prime} e^{i k_{1} \cdot X}\left[X^{I}, X^{J}\right] e^{i k_{2} \cdot X}+O\left(k^{3}\right)
$$

It is also instructive to note that if $\left(k+k^{\prime}+k^{\prime \prime}\right)$ were non-zero in $\hat{O}_{k_{1} k k^{\prime} k^{\prime \prime} k_{2}}$ (open loop), then we could have defined $\hat{O}_{k_{1} k k^{\prime} k^{\prime \prime} p q k_{2}}$ such that $p+q=0$ and $k+k^{\prime}+k^{\prime \prime}+p=0$. This is just 
the same as $\hat{O}_{k_{1} k k^{\prime} k^{\prime \prime} k_{2}}$ but now can be interpreted as a closed loop placed along an open line. Taking the trace would once more move the loop anywhere along the open line. This simple example is illustrative of the fact that in many cases of interest (though not all) open Wilson lines are equivalent to straight Wilson lines with loops attached.

If the loop is not small, Wilson lines of the above type with fixed momentum (Fig. 3) would then seem to correspond to the full closed string in the dual theory. This is the analogue of closed Wilson loop in ordinary gauge theory and reduces to it in the limit of vanishing noncommutativity $(\theta \rightarrow 0)$. It would be interesting to compute the expectation value of the Wilson loop in the large $\theta$ limit using Maldacena duality, much in the same spirit the calculation is done in the absence of the B-field. Also since Wilson loops create electric flux lines, it would be of interest to study the connection with the work of [27] in the case of the $D_{3}$ branes.

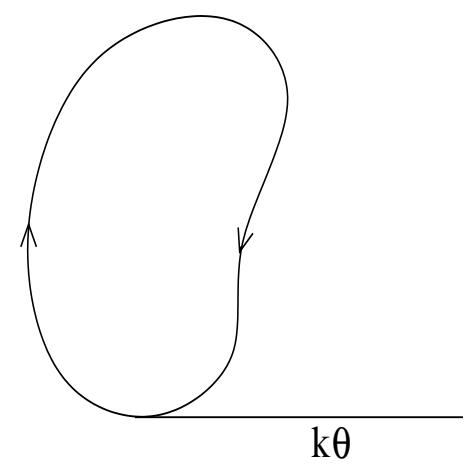

Fig. 3: Large Wilson loop attached to a Wilson line of momentum $k$

\section{References}

[1] N. Seiberg and E. Witten, "Non-commutative Geometry and String theory", JHEP 9909:032 (1999), hep-th/9908142.

[2] J. Maldacena and J. Russo "Large N Limit of Non-Commutative Gauge Theories", hepth/9908134.

[3] A. Hashimoto, N. Itzhaki, "Non-Commutative Yang-Mills and the AdS/CFT Correspondence", hep-th/9907166.

[4] A. Dhar, G. Mandal, S. R. Wadia and K. P. Yogendran, "D1/D5 system with B-field, noncommutative geometry and the CFT of the Higgs branch," hep-th/9910194.

[5] S. Das, S. Kalyana Rama, S. Trivedi, "Supergravity with Self-dual B fields and Instantons in Noncommutative Gauge Theory", hep-th/9911137.

[6] H. Aoki, N. Ishibashi, S. Iso, H. Kawai, Y. Kitazawa, T. Tada, "Noncommutative YangMills in IIB Matrix Model", Nucl.Phys. B565 176 (2000), hep-th/9908141. 
[7] N. Ishibashi, S. Iso, H. Kawai, Y. Kitazawa, "Wilson Loops in Noncommutative Yang Mills", Nucl.Phys. B573 573 (2000), hep-th/9910004.

[8] J. Ambjorn, Y.M. Makeenko, J. Nishimura, R.J. Szabo, "Finite N Matrix Models of Noncommutative Gauge Theory", JHEP 9911 (1999) 029, hep-th/9911041; "Nonperturbative Dynamics of Noncommutative Gauge Theory", Phys.Lett. B480 399 (2000), hep-th/0002158; J. Ambjorn, Y.M. Makeenko, J. Nishimura, R.J. Szabo, "Lattice Gauge Fields and Discrete Noncommutative Yang-Mills Theory", hep-th/0004147.

[9] R. Gopakumar, S. Minwalla, A. Strominger, "Noncommutative Solitons", hepth/0003160.

[10] D. J. Gross and N. Nekrasov, "Monopoles and Strings in Non-Commutative Gauge Theory", hep-th/0005204.

[11] L. Alvarez-gaume and S. R. Wadia, "Gauge Theory on a Quantum Phase Space", hepth/0006219.

[12] J. Maldacena, "Wilson loops in large N field theories", Phys.Rev.Lett. 804859 (1998), hep-th/9803002.

[13] Soo-Jong Rey and R. von Unge, "S-Duality, Noncritical Open String and Noncommutative Gauge Theory", hep-th/0007089.

[14] S. Das and Soo-Jong Rey, "Open Wilson Lines in Noncommutative Gauge Theory and Tomography of Holographic Dual Supergravity", hep-th/0008042.

[15] D. J. Gross, A. Hashimoto, N. Itzhaki, "Observables of Non-Commutative Gauge Theories", hep-th/0008075.

[16] T. Banks, N. Seiberg and S. Shenker, "Branes from Matrices", Nucl. Phys. B490 91 (1997), hep-th/9612157.

[17] M. Li, "Strings from Type II matrices", Nucl. Phys. B499 149 (1997), hep-th/9612222.

[18] A. Fatollahi, "Gauge Symmetry As Symmetry Of Matrix Coordinates", hep-th/0007023.

[19] L. Cornalba and R. Schiappa, "Matrix Theory Star Products from the Born-Infeld Action", hep-th/9907211.

[20] L. Cornalba, "D-brane Physics and Noncommutative Yang-Mills Theory",hepth/9909081.

[21] N. Ishibashi, "A Relation between Commutative and Noncommutative Descriptions of D-branes", hep-th/9909176.

[22] I. Bars and N. Minic, "Non-commutative Geometry on a Discrete Periodic Lattice and Gauge Theory", hep-th/9910091. 
[23] O. Andreev and H. Dorn, "On Open String Sigma-Model and Noncommutative Gauge Fields", hep-th/9912070.

[24] N. Seiberg, "A Note on Background Independence in Noncommutative Gauge Theories, Matrix Model and Tachyon Condensation", hep-th/0008013.

[25] T. Banks, W. Fischler, S.H. Shenker, L. Susskind "M-theory as a Matrix model: a conjecture." Phys. Rev. D55 5112 (1997), hep-th/9610043.

[26] N. Ishibashi, H. Kawai, Y. Kitazawa, A. Tsuchiya "A Large-N Reduced Model as Superstring", hep-th/9612115.

[27] J. Harvey, P. Kraus, F. Larsen and E. Martinec, "D-branes and Strings as Noncommutative Solitons", hep-th/0005031. 